\title{
Stéphane Nahrath et Mathis Stock (dir.), Urbanité et tourisme
}

revue Espaces et Sociétés, nº 151, 2012

\section{Vincent Coëffé}

\section{OpenEdition}

\section{Journals}

\section{Édition électronique}

URL : http://journals.openedition.org/tourisme/165

DOI : 10.4000/tourisme.165

ISSN : 2492-7503

\section{Éditeur}

Éditions touristiques européennes

\section{Édition imprimée}

Date de publication : 1 juin 2014

Pagination : 108-110

ISSN : 2109-5671

\section{Référence électronique}

Vincent Coëffé, «Stéphane Nahrath et Mathis Stock (dir.). Urbanité et tourisme », Mondes du Tourisme [En ligne], 9 | 2014, mis en ligne le 30 septembre 2015, consulté le 22 septembre 2020. URL : http:// journals.openedition.org/tourisme/165 ; DOI : https://doi.org/10.4000/tourisme.165

Ce document a été généré automatiquement le 22 septembre 2020.

\section{(c) () $\odot \odot$}

Mondes du tourisme est mis à disposition selon les termes de la licence Creative Commons Attribution - Pas d'Utilisation Commerciale - Pas de Modification 4.0 International. 


\section{Stéphane Nahrath et Mathis Stock (dir.), Urbanité et tourisme}

revue Espaces et Sociétés, $n^{\circ}$ 151, 2012

\section{Vincent Coëffé}

\section{RÉFÉRENCE}

Stéphane Nahrath et Mathis Stock (dir.), Urbanité et tourisme (dossier), Revue Espaces et Sociétés, $\mathrm{n}^{\mathrm{o}} 151,2012$.

1 Ce dossier de la revue Espaces et Sociétés offre un "espace public" à un thème encore trop peu visité, celui des relations entre urbanité et tourisme. L'habitude a en effet été prise dans le segment scientifique comme dans la "société civile" de multiplier les adjonctions d'épithètes au terme tourisme, jusqu'à produire la formule rarement déconstruite de "tourisme urbain", souvent mobilisée pour traiter en réalité du tourisme dans la ville, comme cela avait été souligné dans l'ouvrage dirigé en 2007 par Philippe Duhamel et Rémy Knafou sur Les Mondes urbains du tourisme.

2 Ce dossier, en prenant le parti de parler d'urbanité, cherche à poursuivre le travail théorique engagé sur l'objet "tourisme", qui ne peut plus être seulement défini en extension comme nous l'avons déjà souligné, mais qui gagnerait désormais à être défini en compréhension par l'analyse de ses propriétés. La conceptualisation va dans ce sens, et l'urbanité émerge comme un outil qui permettrait de sortir du piège de la formule laissant penser que l'on pourrait dresser des lignes de partage entre, d'un côté, un "tourisme urbain" et, de l'autre, d'autres formes supposées de tourisme, tantôt qualifié de "rural", de "balnéaire", voire de "vert", "blanc", "noir", "rouge", etc. Comme si, autrement dit, le tourisme dit "balnéaire" (par exemple) était découplé de l'urbain.

Or, comme le précisent Stéphane Nahrath et Mathis Stock dans leur éditorial intitulé "Urbanité et tourisme: une relation à repenser", "l'urbanisation et la touristification constituent depuis plus d'un siècle deux processus majeurs de développement de sociétés 
occidentales, puis mondiales, qui entretiennent des relations à la fois d'interdépendance et de tensions. Ces deux processus s'informent réciproquement au sens où l'urbain est fondamentalement à l'œuvre dans le touristique, et le touristique fait émerger de nouvelles formes et normes urbaines".

L'avantage du concept d'urbanité est qu'il renvoie à l'idée de "qualité de l'urbain, telle qu'elle se manifeste à la fois dans l'organisation spatiale (concentrations, polarités, centralités, couplage entre diversité et densité des réalités sociétales), mais également dans le rapport à l'urbain tel qu'il se manifeste dans les phénomènes d'identité, d'altérité, de la 'civil inattention' ou encore de l'attention à l'autre".

Cette proposition théorique, qui est aussi programmatique pour le dossier d'Espaces et Sociétés, est plus ou moins mise en œuvre dans les cinq articles qui composent la livraison. Cela dit, comme l'indique l'éditorial lui-même, le dossier est surtout organisé autour de la proposition de Mathis Stock et Léopold Lucas "concernant l'évolution historique des rapports entre urbanité et tourisme". Les deux auteurs ont en effet fait le choix de mettre au jour, dans leur article intitulé "La double révolution urbaine du tourisme", "deux grandes formes historiques (successives) de relations entre urbanisation et touristification".

6 La première serait mise en place à partir de la fin $\mathrm{du}_{\mathrm{XVIII}}{ }^{e}$ siècle et correspondrait à la diffusion du tourisme, qui enclencherait l'urbanisation de "lieux périphériques", jusqu'à propulser ces derniers dans un "espace d'urbanité international". Cette "révolution", terme que nous pourrions interpréter ici comme renvoyant à l'univers théorique d'Henry Lefebvre (et à son œuvre majeure, La Révolution urbaine), voit l'apparition de la "station", un type de lieu touristique qui est régulièrement écarté de l'analyse urbaine au prétexte que sa masse démographique ne serait pas celle d'une ville. Or, les stations sont produites, en même temps qu'elles les produisent, par certains attributs urbains, lesquels ne peuvent plus être seulement mobilisés pour analyser la ville dans un contexte d'urbanisation généralisée (Lussault, 2007 ; 2013). Du coup, les stations, lieux habités surtout par des touristes qui sont "fondamentalement des citadins [contribuant] à transformer l'ensemble des lieux pratiqués en lieux urbains", peuvent être analysées comme des agencements singuliers d'ingrédients urbains parmi lesquels figurent la densité, la diversité, la centralité et l'espace public, surtout si ce dernier est conçu comme l'espace de l'anonymat, de l'imprévu, et de la mise à l'épreuve des civilités (Coëffé, 2010). Ces dernières dimensions restent toutefois assez discrètes dans cette livraison et montrent que la "qualité urbaine" du tourisme peine encore à passer par l'analyse de pratiques informées par l'actualisation des normes.

7 S'ils ne traitent pas spécifiquement de la station touristique, Pierre-Olaf Schut et Éric Levet-Labry, dans leur article intitulé "Le rôle des pratiques sportives et de loisirs dans la définition et l'urbanisation des sites touristiques en France", montrent ainsi comment la diffusion du tourisme dit "sportif" peut passer par les "sociétés d'encouragement touristiques" qui "mobilisent les acteurs publics et privés pour offrir les conditions optimales au développement du tourisme sur le territoire, en facilitant l'exploration et en réduisant les risques objectifs liés à la pratique". Or, dans cette dynamique, les "infrastructures, les moyens de communication et les services mis en place contribuent à propager des traits d'urbanité dans des campagnes plus ou moins marquées par l'isolement et la désertification. Il s'agit de former les autochtones à l'accueil pour que les touristes puissent retrouver leurs repères de citadins, tout en ayant l'impression de participer à une exploration". 
De son côté, Marie-Ève Férérol, dans son article intitulé "Naissance et développement de La Bourboule : ville thermale neuve française exemplaire", bouscule la nomenclature urbaine, en qualifiant de ville un lieu régulièrement identifié en tant que "station". L'itinéraire du lieu (Équipe Mit, 2011) montre une mise en tourisme rapide à partir de la fin du xix $x^{e}$ siècle et le début du $x^{e}$ siècle, fondée notamment sur une "alliance loisirsmédecine": "D'un simple hameau, La Bourboule est devenue, en un temps record (30 ans) une ville au plan quadrillé, parsemée de remarquables édifices dont la plupart sont l'ouvre d'architectes de renom. Le tourisme est apparu comme un moyen de s'affranchir de la commune voisine. Émancipée, elle a su capter au fil des années les principaux équipements qui font d'elle [...] le centre d'un espace touristique plus large (le massif du Sancy) et un pôle de centralité." Ainsi, de "localité monofonctionnelle et saisonnière, elle présente aujourd'hui plusieurs facettes urbaines : résidentielle, commerciale, administrative et touristique (tourisme de congrès, sportif, etc.)".

9 La seconde "révolution" serait lancée durant la seconde moitié du $x^{e}$ siècle, et correspondrait à l'investissement des centres de certaines villes (y compris des métropoles) par le tourisme, processus qui participerait parfois d'une reconfiguration profonde de ces lieux. Alors que le tourisme pouvait constituer une "activité économique parmi d'autres, longtemps minoritaire même, il devient un élément de plus en plus déterminant dans la constitution de l'urbanité de ces lieux, au point que l'on peut avancer l'idée d'un recreational turn (Stock, 2006) pour les villes européennes dans un premier temps et maintenant observable à travers le monde [...]".

Ce recreational turn s'observe par exemple à Paris à travers l'opération Paris Plages analysée de manière assez originale par Benjamin Pradel et Gwendal Simon. "Plage éphémère" installée sur la voie Georges-Pompidou au centre de la capitale "depuis dix ans, de la mi-juillet à la mi-août", Paris Plages peut être analysé comme un dispositif articulant une "césure spatiale par l'aménagement temporaire d'un espace public clos et une césure symbolique par l'utilisation de signifiants balnéaires qui instrumentalisent l'imaginaire touristique des vacances estivales en bord de mer. La mise en scène du cadre urbain quotidien repose sur la mobilisation de la sémiosphère touristique balnéaire qui, tel 'un jeu de mécano', est censée "permettre aux gens de croire à l'espace et donc de se mettre en maillot de bain". En injectant du hors-quotidien et de l'ailleurs dans la métropole, Paris Plages n'est donc pas qu'une scénographie qui fonctionnerait comme un simple décor. Le dispositif, dans un jeu subtil de distance/proximité au réel, offre des prises aux individus afin que ces derniers négocient leurs actions par rapport au contexte qu'ils tendent à produire en retour. La question des convenances est donc redéfinie ici selon de nouvelles modalités qui autorisent par exemple les "plageurs" (Urbain, 1994) à endosser un costume extraordinaire (par rapport au contexte qui domine une fois l'événement achevé) mais conforme à une nouvelle configuration spatiale et sociale: un simulacre de la balnéarité. Mais Paris Plages permet de révéler, selon les auteurs, une hybridation des pratiques, démonstration qui valide en partie la proposition énoncée par Michel Lussault, selon qui "le quotidien s'hybride du hors-quotidien et réciproquement. Le tourisme travaille la quotidienneté qui s'avère informée, voire saturée d'informations par la sémiosphère touristique, liée à la pratique effective de l'activité mais aussi à sa mise en public" (2007). De fait, Paris Plages n'a pas été conçu a priori comme un événement créant les conditions d'une "mise en tourisme". Si la scénographie mobilise des référents associés à un certain univers touristique, les pratiques montrent le "comportement hybride d'un citadin qui joue au plagiste. La distance au quotidien assumée par le plagiste urbain s'incarne en partie 
dans le rapport au corps inédit qu'il pratique en ville et qui est travaillé par le voisinage de ses semblables [...]. Le maillot de bain devient un élément de légitimation du dévoilement des corps autant que la validation de la scénographie. [...] Cependant, la ville se rappelle au bon souvenir de chacun puisque le monokini, bikini et nudisme sont interdits par le règlement intérieur et que la foule qui défile devant les plages limite les pratiques trop ostentatoires. [...] Le statut du visiteur sur le sable oscille ainsi entre plagiste et citadin".

11 Autre article original, celui d'Émilie Flon, intitulé "De la mise en scène à la fiction touristique : la médiation du voyage urbain sur la ligne de métro Xinbeitou à Taipei". L'originalité apparaît dès les premières lignes de l'introduction puisque l'auteur rappelle combien la mobilité constitue non pas un résidu de l'urbanité mais une de ses propriétés: “Ce qui se produit dans les espaces et les temps de la mobilité n'est pas un simple effet secondaire de l'urbain, mais participe plutôt à caractériser l'urbanité, la ville en tant qu'elle est ville, y compris ses différents modèles politiques." L'auteur s'appuie notamment sur le dispositif théorique de Georges Amar, lequel renverse la "primauté conceptuelle" attribuée ordinairement au lieu sur le mouvement, comme si ce dernier n'était qu'un simple intervalle à réduire entre deux points déjà là, a priori constitués, et sans que le mouvement joue dans leur production elle-même.

Il est vrai aussi que la singularité de l'article tient dans l'objet même que l'auteur analyse. Car s'il s'agit de pointer la focale sur "l'aménagement d'un mode de transport qui paraît renouveler cette acception du mouvement urbain, [...] l'originalité de la ligne de métro Xinbeitou à Taipei est de se constituer également en un dispositif touristique: les rames intègrent une ambiance et une mise en scène de la pratique touristique urbaine qui présentent de nombreuses similitudes avec certaines expositions culturelles, proposant ainsi une activité à ses voyageurs dans un lieu et un temps habituellement coupés de la ville et plutôt consacrés à l'attente".

\section{BIBLIOGRAPHIE}

Vincent COËFFÉ, "Le tourisme, fabrique d'urbanité. Matériaux pour une théorie de l'urbain", Mondes du ourisme, $\mathrm{n}^{\circ} 2,2010$.

Michel Lussault, "Le tourisme, un genre commun", dans Philippe Duhamel et Rémy Knafou (dir.), Mondes urbains du tourisme, Belin, 2007.

Michel Lussault, “Urbanité”, Dictionnaire de la géographie et de l'espace des sociétés, Armand Colin, 2013.

Équipe MIT, Tourismes 3. La révolution durable, Belin, 2011.

Mathis sтоск, "European cities: Towards a recreational turn?", Hagar. Studies in Culture, Polity and Identities, vol. 7, $\mathrm{n}^{\circ} 1,2006$.

Jean-Didier URBAIN, Sur la plage. Mœurs et coutumes balnéaires, Payot, 1994. 
AUTEURS

VINCENT COËFFÉ

Université d'Angers [vincent.coeffe@univ-angers.fr] 